\title{
Challenges and Opportunities of Psychiatric Training During COVID-19: Early Career Psychiatrists' Perspective Across the World
}

\author{
Sachin Nagendrappa ${ }^{1} \cdot$ Renato de Filippis $^{2}$ (D) $\cdot$ Rodrigo Ramalho $^{3} \cdot$ Ramdas Ransing $^{4} \cdot$ Laura Orsolini $^{5} \cdot$ Irfan Ullah $^{6}$. \\ Ruta Karaliuniene $^{7}$. Sheikh Shoib ${ }^{8} \cdot$ Zargham Abbass $^{9} \cdot$ Nafisatu Hayatudeen $^{10} \cdot$ Chonnakarn Jatchavala $^{11}$. \\ Mariana Pinto da Costa ${ }^{12} \cdot$ Victor Pereira-Sanchez $^{13}$
}

Received: 15 January 2021 / Accepted: 6 May 2021 / Published online: 25 May 2021

(C) Academic Psychiatry 2021

\section{To the Editor:}

The coronavirus pandemic and subsequent lockdown measures led to logistical difficulties that prevented numerous psychiatric patients from accessing hospital-based treatment. Many trainees were redeployed to specialized COVID-19 units to provide general health services for people with COVID-19. In this and other ways, the pandemic has affected the education of postgraduate trainees in several medical specialties, including psychiatry, throughout the world [1].

The lockdown measures forced inpatient and outpatient psychiatric services to significantly reduce their clinical capacity and limit access to mental health services. These adjustments impacted face-to-face interactions, thereby limiting educational, supervision, and mentorship activities for trainees. Resources dedicated to research and to enrolling patients in clinical trials were also reduced. At the same time, many psychiatric trainees and consultant psychiatrists were redeployed to non-psychiatric wards, due to the lack of other medical staff, effectively interrupting regularly scheduled training activities [2]. Thus, the primary objective of tackling

Renato de Filippis

defilippisrenato@gmail.com

1 National Institute of Mental Health and Neurosciences (NIMHANS), Bengaluru, India

2 University Magna Graecia of Catanzaro, Catanzaro, Italy

3 The University of Auckland, Auckland, New Zealand

4 BKL Walawalkar Rural Medical College, Ratnagiri, Maharashtra, India

5 Polytechnic University of Marche, Ancona, Italy

6 Gandhara University, Peshawar, Pakistan the pandemic often relegated academic training to a secondary, sometimes neglected, role [3].

These changes have affected training programs in psychiatry [4]. In place of regular in-person teaching, training programs, meetings, and patient encounters were shifted to online platforms [5]. Postgraduate courses, conferences, and workshops were made virtual, giving many trainees the opportunity to attend international events. Individual training institutions moved many of their regular didactic programs and journal clubs online. As some of these changes may endure past the pandemic, their value will need to be reassessed in the future.

As early career mental health clinicians and researchers from different countries, we, members of the Global Mental Health Think Tank, connected through the Early Career Psychiatrists' Section of the World Psychiatric Association, have identified five core areas of psychiatric training affected worldwide by the COVID-19 pandemic:

- Quality of training: The workload, including nonpsychiatric care responsibilities, for which many trainees had minimal training, has increased dramatically. As

7 Technical University Dresden, Dresden, Germany

8 Jawahar Lal Nehru Memorial Hospital (JLNMH), Srinagar, Jammu and Kashmir, India

9 University of Missouri - Kansas City (UMKC), Kansas City, MO, USA

10 Federal Neuropsychiatric Hospital, Kaduna, Nigeria

11 Prince of Songkla University, Hat Yai, Thailand

12 Institute of Psychiatry, Psychology \& Neuroscience, King's College London, London, UK

13 NYU Grossman School of Medicine, New York, NY, USA 
clinical staff has been meeting these increased demands and attention and resources in medical centers have been focused on COVID-related activities, training programs have found it challenging to maintain standards of high quality in their theoretical and practical didactic activities aiming at enriching trainees' knowledge and clinical skills as future psychiatrists.

- Lack of diversified clinical exposure and practice: At times, service needs have been prioritized over training. In some countries, trainees were instructed not to rotate to a new placement to guarantee continuity of service provision. Also, despite population-wide increases in psychological distress, some trainees have experienced reductions in patient caseloads during lockdowns, especially in outpatient clinics and multidisciplinary collaborative care. The closure of outpatient departments and logistical difficulties accessing hospitals were contributing factors for this decrease of clinical exposure. This has been somewhat balanced by patients having access to traineeprovided care through telehealth.

- Lack of research activities: Research activities, usually optional in training, have become even more limited during the pandemic for many trainees. For all clinical studies, recruiting suitable patients during the pandemic has become even more difficult. Therefore, early-career investigators at all levels have found it difficult to submit their theses, dissertations, and research projects within the previously estimated time frames, follow their study protocols, or attain their previously expected subject accruals.

- Use of technology for education: The implementations of telepsychiatry and tele-education have accelerated worldwide. This was in response to the sudden impossibility of meeting in-person and to complement the fewer in-person opportunities for learning. These approaches may continue to offer benefits beyond the pandemic. Trainees have been so far supported by moving meetings online and allowing for the continuity of virtual learning through webinars and e-learning modules.

- Effects on entrance and exit exams: With the changes in clinical practice, physical distancing rules, and travel restrictions, assessments became challenging to complete. Most exams were delayed, and others cancelled.

In response to these challenges, measures have been taken to help trainees and trainers in psychiatry during these difficult times. Indeed, telemental health and education have enhanced alternative ways of psychiatric training during the COVID-19 pandemic. Training committees have put forth various recommendations, including closer supervision, scheduled assessments, extending deadlines for submission of dissertations/ projects, rotations across subspecialties, including research training, and peer-support groups. Other priorities have included providing trainees with adequate support regarding life-work balance to manage the added stress and workload brought on by COVID-19 and to decrease the risk of burnout.

Possibly the key lesson for students, trainers, and trainees has been the need to be flexible and adaptable to different situations, skills that will make them better psychiatrists in the future.

It remains important to evaluate, during the post-peak wave or post-pandemic period, whether it will be best to return to traditional learning methods or to adopt a blend of in-person and virtual teaching and learning approaches. It will also be necessary to assess whether postgraduate medical training programs have achieved the main learning outcomes established for psychiatric trainees during their psychiatric training programs or if their learning has been negatively affected by the current COVID-19 circumstances, and to what extent. Therefore, it is increasingly important to implement measures that ensure an adequate level of training even during the pandemic. The world has already paid a high price during the COVID-19 pandemic, but there is hope that we can limit and reduce its damage, especially concerning education.

Acknowledgements The authors would like to acknowledge the Early Career Psychiatrists (ECPs) Section of the World Psychiatric Association (WPA) and the Global Mental Health Think Tank. We are thankful to Francisco X. Castellanos, MD, for proofreading our manuscript draft and providing suggestions.

\section{Declarations}

Disclosures On behalf of all authors, the corresponding author states that there is no conflict of interest.

\section{References}

1. Woolliscroft JO. Innovation in response to the COVID-19 pandemic crisis. Acad Med. 2020;95:1140-2.

2. Pereira-Sanchez V, Adiukwu F, El Hayek S, Bytyçi DG, GonzalezDiaz JM, Kundadak GK, et al. COVID-19 effect on mental health: patients and workforce. Lancet Psychiatry. 2020;7:e29-30.

3. Singh K, Srivastav S, Bhardwaj A, Dixit A, Misra S. Medical education during the COVID-19 pandemic: a single institution experience. Indian Pediatr. 2020;57:678-9.

4. Low JCM, Visagan R, Perera A. Neurosurgical training during COVID-19 pandemic: British perspective. World Neurosurg. 2020;142:520-2.

5. Schuh Teixeira AL, Spadini AV, Pereira-Sanchez V, Ojeahere MI, Morimoto K, Chang A, et al. The urge to implement and expand telepsychiatry during the COVID-19 crisis: early career psychiatrists' perspective. Rev Psiquiatr Salud Mental. 2020;13:174-5.

Publisher's Note Springer Nature remains neutral with regard to jurisdictional claims in published maps and institutional affiliations. 